\title{
CENTER OF EXCELLENCE for Education, Science \& Technology in 21 Century BIS-21
}

Tn 2000, the Central Laboratory for Parallel Processing (CLPP) of the Bulgarian Academy of Sciences has been recognized as a Center of Excellence for learning, scholarship and research in the Eastern-European region. It has a leading position among the scientific institutions in Bulgaria in the fields of Computer Science, Scientific Computations, Supercomputing, Linguistic Modelling and Communications and Control. It performs research, consultations, projects and high quality education in its fields of interest. The activities of CLPP are oriented mainly to the creation and usage of advanced mathematical and computer technologies. During the last years fundamental results in different areas of the Theoretical Informatics and Scientific Computations were obtained. They were motivated by the developed by the Laboratory staff methods, algorithms and software as well as by introduced advanced information technology and computer facilities. Nowadays, the scientific strategy of CLPP consists in the development of multiprocessors systems and new highperformance algorithms for supercomputer (vector and/or parallel computers with shared and distributed memory, including here clusters of workstations) The results obtained by Laboratory staff are applied in various fields - ecology, engineering, computer technology, information systems, etc. The CLPP Computer Center maintains the information network of the Bulgarian Academy of Sciences. Annually, scientists from the Laboratory publish about 140 papers, and about 100 are in refereed international journals and proceedings of highly recognized international conferences.

\section{History}

The Central Laboratory for Parallel Processing was established in 1985. It was founded as Center for Informatics and Computer Technology (CICT) by group of scientists headed by acad. Blagovest Sendov who was the first Director of this institution. The main idea was to coordinate research in the field of Computer Science and Compute Technology done by scientists from Bulgarian Academy of Sciences, Bulgarian Universities and some Institutes belong to the Industry. In a short time 
many senior scientists as well as young mathematicians, engineers and computer science specialists were attached to the group and in only one year CICT took a leading position in Bulgaria in the field of Computer Science and Computer Technology. In 1996 CICT was renamed as Central Laboratory for Parallel Processing. CLPP has been, and still is, an active participant in a number of research and educational projects of the EU programs INCO-COPERNICUS, TEMPUS, PECO, GO EAST GO WEST, etc. as well as in NATO Scientific Programs. A lot of the scientists of the Laboratory has been on long-term specializations in USA, UK, Denmark, France, Germany, the Netherlands, etc. CLPP has organized many international conferences and workshops. Some of them are periodical as Parallel and Distributed Processing, Network Information Processing Systems,

\section{Structure}

CLPP is headed by a Director, Deputy Director and Scientific Secretary. General and scientific police of the Laboratory is formulated by the Directors Board including all heads of Departments and 24-members Scientific Council. Members of the Scientific Council are most of the full and associate professors of CLPP as well as some of most famous scientists in the fields of interest from other Institutes of the Bulgarian Academy of Sciences. CLPP consists of six Departments: Distributed Computing Systems and Networks, Parallel Algorithms, Scientific Computing, High-Performance Computer Architectures, Linguistic Modelling, Mathematical Methods for Sensor Information Processing and Computer Center. CLPP disposes of the following computer facilities: CRAY ORIGIN 2000, IBM 4381 with 65 local terminals, VAX 3100 with 24 terminals, at about 45 PC's AT 486 and 586, 18 SUN Microsystems workstations including one 4-processors stations, SGI-O2 station, HP 9000-C class. All of them are connected through a local LAN Ethernet to the International network.

\section{Staff}

The total staff of CLPP is 112 persons including one academician, one corresponding member of the Academy, six full professors, 24 associate professors, 39 research assistants and 20 specialists with university education. Every year students from Sofia University (Faculty of Mathematics and Informatics) and the Technical University of Sofia prepare their MSc theses in the Laboratory having as supervisors Senior Scientists from the staff. Permanently there are five-six PhD students in the field of Numerical Mathematics, Computer Science and Computer Technology and their applications. Most of the Senior Scientists of CLPP are affiliated with various national and international administrative organizations such as the National Research Fund, the Higher Evaluation Commission, the Executive Council of the Bulgarian Academy of Sciences, AMS, British Computer Society, ISCU, IMU, Int. Commission 
for Math. Education, Green Gross, etc. Many full and associate professors, as well as some of the research associates, lecture on their fields of interest in several Universities in Sofia, Plovdiv, Varna, Bourgas and Blagoevgrad for undergraduate, graduate and postgraduate students. 